\title{
Mesophase Behaviour of a Cyanobiphenyl Molecule in Polar Aprotic Solvent: Rigidity Effect
}

\author{
Suraj Kumar Nayak and P. Lakshmi Praveen* \\ Department of Physics, Veer Surendra Sai University of Technology, \\ Burla-768018, Sambalpur, Odisha, India \\ *Corresponding author: plpraveen_phy@vssut.ac.in
}

Published online: 25 November 2020

To cite this article: Nayak, S. K. \& Praveen, P. L. (2020). Mesophase behaviour of a cyanobiphenyl molecule in polar aprotic solvent: Rigidity effect. J. Phys. Sci., 31(3), 33-45. https://doi.org/10.21315/jps2020.31.3.3

To link to this article: https://doi.org/10.21315/jps2020.31.3.3

\begin{abstract}
In this paper, a theoretical study has been carried out on a liquid crystal compound named p-n-propyl cyanobiphenyl (3CB). The different modes of interaction energy values in a polar aprotic solvent (ethyl acetate) for small amount of translation and rotation are calculated. The corresponding probabilities have been calculated at both room temperature $(300 \mathrm{~K})$ and transition temperature $(303.3 \mathrm{~K})$. The rigidity parameter for stacking and in-plane interactions has been estimated and then the stability of molecule according to probability and rigidity at definite translation and rotation has been concluded. The change in the characteristics and stability of the compound at transition temperature has been observed. The dependence of mesophase behaviour with change in the certain configurations and orientation of the molecules have been discussed. These observed results provide an insight about the process of mesophase structure and its formation. The present compound may guide in establishing the other molecular models with transition temperature nearer to room temperature.
\end{abstract}

Keywords: Liquid crystal, 3CB, mesophase, rigidity, cyanobiphenyl molecule

\section{INTRODUCTION}

In liquid crystal phase, the molecules are with enough disorder that offers flow properties, and some degree of ordering that leads to anisotropy. ${ }^{1-4}$ This phase offers higher degree of orientational order to a liquid and a less positional order to a crystal. The molecules ordering in a liquid crystal phase may be either translational and/or rotational. The translational order can be realised with respect to the atoms position in a space in a regular array. ${ }^{5,6}$ Further, the rotational order is 
considerable for the non-spherical molecules. To analyse the mesophase behaviour of a compound theoretically, a quantum mechanical approach is suitable. ${ }^{7-9}$ The analysis of mesophase behaviour requires the energy values of a configuration of the molecule corresponding to each translation and rotation. This estimation requires an analysis of both the faces, sides of a molecule and the energy values of a configuration have to be calculated. Further, the probability of each configuration is required in order to compare among the sides, faces and to obtain the most stable configuration. An adequate quantum chemical approach is essential for a reasonable sketch of the structure that includes the probabilities of configurations with respect to different molecular motions. The popular semi-empirical method for this task is complete neglect of differential overlap (CNDO/2), established by Pople and Beveridge. ${ }^{10}$ But the molecular pair interaction offers better analysis on behaviour of a mesogens.

The interactions between a molecular pair of mesogens, anisotropy of pair potential, configuration with minimum energy, and the subsequent parameters can be achieved through these analyses. Further, multipole interactions offer better description of considerable variations among the energies of configurations. ${ }^{11,12}$ The estimated energy differences play a vital role to judge the phase behaviour of the compounds. Therefore, relative energies are useful to study the probability of configurations. ${ }^{13}$ Hence, in the present paper, the authors report the interaction energy, and configurational probability of a nematogen $p$ - $n$-propyl cyanobiphenyl $(3 \mathrm{CB})$ in a polar aprotic solvent (ethyl acetate) at room temperature $(300 \mathrm{~K})$ and nematic-isotropic transition temperature $(303.3 \mathrm{~K}) .{ }^{14}$ The self-assembly nature of nematic mesogens is helpful for superior applications. ${ }^{15,16}$ Configurational probabilities of a molecular pair have been computed at an intermediate distance $\pm 6 \AA$ for stacking and $\pm 8 \AA$ for in-plane interactions. The choice of distance has been made to eliminate the possibility of van der Waals contacts completely, and to keep the molecules within the short- and medium-range interactions.

\section{METHOD}

The molecular geometry of $3 \mathrm{CB}$ has been constructed on the basis of published crystallographic data with the standard values of bond lengths and bond angles. ${ }^{14}$ The following computations have been carried out:

\subsection{Computation of Atomic Net Charge and Dipole Moments}

In the present computation, the $\mathrm{CNDO} / 2$ method has been employed to compute the net atomic charge and dipole moment at each atomic centre of the molecule. ${ }^{10}$ 


\subsection{Computation of Interaction Energy at Various Configurations}

A detailed computational scheme based on simplified formula by Claverie for the evaluation of interaction energy between a molecular pair has been used to calculate the energy for fixed configuration. ${ }^{17}$ According to the second order perturbation theory for intermediate range interactions, the total pair interaction energy of molecules $\left(U_{\text {pair }}\right)$ is represented as sum of various terms contributing to the total energy: ${ }^{18}$

$$
U_{\text {pair }}=U_{e l}+U_{p o l}+U_{\text {disp }}+U_{\text {rep }}
$$

where $U_{e l}, U_{p o l}, U_{\text {disp }}$ and $U_{\text {rep }}$ are the electrostatic, polarisation, dispersion and repulsion energy terms, respectively. Again, electrostatic term is expressed as:

$$
U_{e l}=U_{Q Q}+U_{Q M I}+U_{M I M I}+\ldots
$$

where $U_{Q Q}, U_{Q M I}$ and $U_{M I M I}$, etc., are monopole-monopole, monopole-dipole and dipole-dipole terms, respectively. In fact, the inclusion of higher order multipoles does not significantly affect the electrostatic interaction energy and the calculation only up to dipole-dipole term gives satisfactory result. The computation of electrostatic term has, therefore, been restricted only up to dipole-dipole energy term. In the present computation, the dispersion and short-range repulsion terms are considered together because the several semiempirical approach, viz. the Lennard-Jones or Buckingham type approach, actually proceed in this way. Kitaygorodsky introduced a Buckingham formula whose parameters were later modified for hydrocarbon molecules and the several other molecules and finally gave the expression: ${ }^{19,20}$

$$
\begin{aligned}
& U_{\text {disp }}+U_{\text {rep }}=\sum_{\lambda}^{(1)} \sum_{v}^{(2)} U(\lambda, v) \\
& U(\lambda, v)=K_{\lambda} K_{v}\left(-A / Z^{6}+B e^{-\gamma \mathrm{Z}}\right)
\end{aligned}
$$

where $Z=R_{\lambda v} / R_{\lambda v}^{0} ; R_{\lambda v}^{0}=\left[\left(2 R_{\lambda}^{w}\right)\left(2 R^{w}{ }_{v}\right)\right]^{1 / 2}$, in which $R^{w}{ }_{\lambda}$ and $R^{w}{ }_{v}$ are the van der Waals radii of atom $\lambda$ and $v$, respectively. The parameters $A, B$ and $\gamma$ do not depend on the atomic species. But $R_{\lambda v}^{0}$ and factor $K_{\lambda} K_{v}$ allow the energy minimum to have different values according to the atomic species involved. 


\subsection{Computation of Configurational Probabilities}

The total interaction energy values obtained through these computations have been used as an input to calculate the probability of occurrence of a particular configuration $i$ using the Maxwell-Boltzmann formula in order to obtain a better insight: ${ }^{21}$

$$
P_{i}=\exp \left(-\beta \varepsilon_{i}\right) / \Sigma_{i} \exp \left(-\beta \varepsilon_{i}\right)
$$

where $P_{i}$ stands for probability, $\beta=1 / k T, k$ is the Boltzmann constant, $T$ is the absolute temperature and $\varepsilon_{i}$ represents the energy of the configuration $i$ to the minimum energy value in a particular set for which the probability distribution is computed. An orthogonal coordinate system has been considered to facilitate the above calculation. The origin has been chosen at almost midpoint of the molecule. The $\mathrm{x}$-axis has been chosen along a bond parallel to the long molecular axis while the $y$-axis lies in the plane of the molecule and z-axis is perpendicular to the $x-y$ plane.

\section{RESULTS AND DISCUSSION}

The structure of 3CB molecule is shown in Figure 1. The arrangement of molecular pair during different modes of interactions is shown in Figure 2. The results of different interaction modes are discussed below.

\subsection{Interaction Energy in Stacking Mode}

The associating molecules are taken and constrained in the coordinate system in such a way that the long axis of the molecule is directed parallel to the $\mathrm{x}$-axis and another molecule in the z-axis at interspace of $\pm 6 \AA$ from the constrained molecule. The molecule is now rotated about $\mathrm{x}$-axis and the alteration of total energy is carried out on face $1\left(\mathrm{~F}_{1}\right)$ and face $2\left(\mathrm{~F}_{2}\right)$ of the $3 \mathrm{CB}$ molecules for the configuration y $\left(0^{\circ}\right) \mathrm{z}\left(0^{\circ}\right)$. Configuration $\mathrm{y}\left(\theta_{1}^{\circ}\right) \mathrm{z}\left(\theta_{2}^{\circ}\right)$ means that the molecule has been rotated from its initial position by $\theta_{1}{ }^{\circ}$ about y-axis and by $\theta_{2}{ }^{\circ}$ about z-axis. The total interaction energy is found to be nearly same for both faces. The molecule is then shifted to finite distance from the chosen initial position and calculations of interaction energy have been done. The attained minimum energy is set as an initial value and this procedure is further reiterated for smaller intervals. This is done by keeping all axes along the long axis of the molecule in turns. Translations and rotations have been made about all the axes to minimise the energy. During this procedure, $0.1 \AA$ of accuracy in translation and about $1^{\circ}$ in rotation is attained for a molecule with respect to other molecules via auto process. 


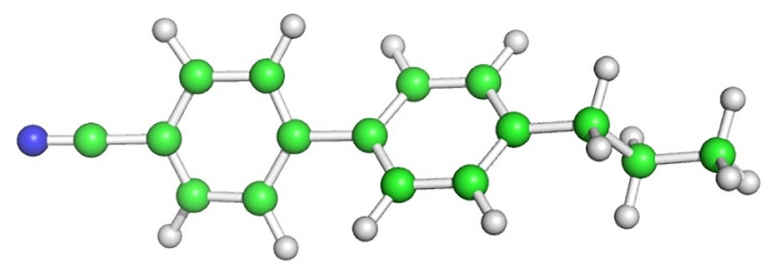

Figure 1: Structure of 3CB molecule.

(a)

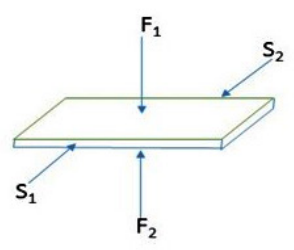

(c)

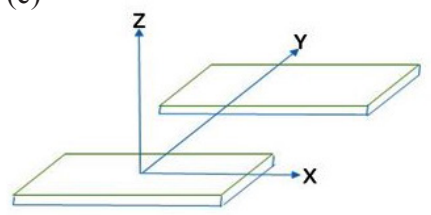

(b)

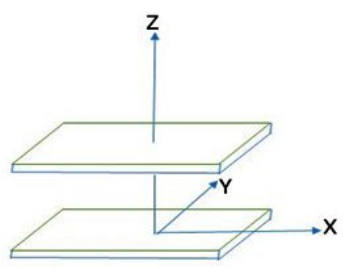

(d)

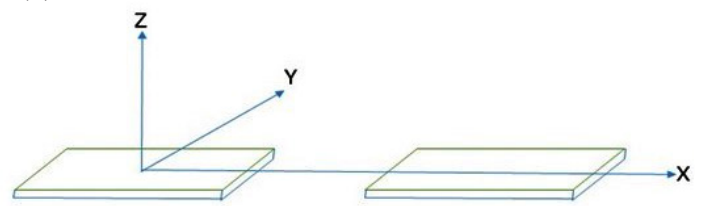

Figure 2: Illustrations of (a) faces $\mathrm{F}_{1}, \mathrm{~F}_{2}$ and sides $\mathrm{S}_{1}, \mathrm{~S}_{2}$ of a molecule, (b) stacking interaction, (c) in-plane interaction, and (d) terminal interaction.

Further, the study of the total interaction energy is carried out for both faces of $3 \mathrm{CB}$ molecules during translation along the long axis (x-axis) of the molecule for the configuration $\mathrm{y}\left(0^{\circ}\right) \mathrm{z}\left(180^{\circ}\right)$ as shown in Figure 3 . The energy minima for faces 1 and 2 are found to be $-1.1997 \mathrm{kcal} \mathrm{mole}^{-1}$ and $-1.3983 \mathrm{kcal} \mathrm{mole}^{-1}$, respectively. It has been observed that $F_{1}$ and $F_{2}$ have an aligned structural preference. The stacked molecules can glide over one another in the range $0 \pm 6 \AA$ without any change in energy. At the disposal of thermal perturbation these molecules are found to have a good stability and are capable of retaining a molecular order up to $30 \AA$. 


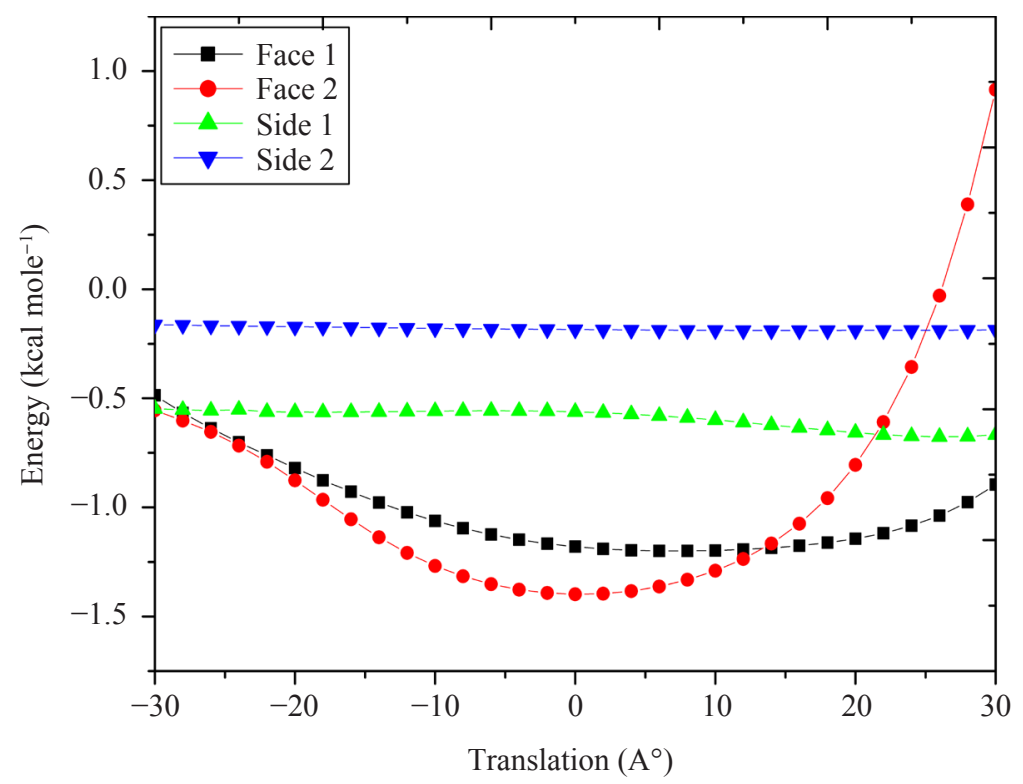

Figure 3: Variation of total stacking (face 1,2) and in-plane (side 1,2) interaction energy of $3 \mathrm{CB}$ with respect to translation along $\mathrm{x}$-axis for the configuration $\mathrm{y}\left(0^{\circ}\right)$ $\mathrm{z}\left(180^{\circ}\right)$ and $\mathrm{y}\left(0^{\circ}\right)$, respectively.

\subsection{Interaction Energy in In-plane Mode}

The associating molecules are taken and constrained in the coordinate system and fixed along the y-axis. This distance is maintained to avert the van der Waal contacts. Similar computations are done for both the $1\left(\mathrm{~S}_{1}\right)$ and $2\left(\mathrm{~S}_{2}\right)$. The molecules were rotated and translated slowly and minutely about the co-ordinate axes for the minimisation of energy in a selected configuration of the associating molecules. When the configuration is changed, i.e., the molecules were rotated about $\mathrm{x}$-axis and $\mathrm{y}\left(0^{\circ}\right)$ configuration was set and the alteration of total interaction energy was carried out for both sides $\mathrm{S}_{1}$ and $\mathrm{S}_{2}$, then a clear preference in structure was observed though the energy difference was very small for different rotation angles. Hence, generally it may be assumed that when the molecules are in the same plane then at low temperature the molecules could rotate freely in in-plane interactions.

The nematic behaviour is characterised from the translational freedom of the molecule. The study of total interaction energy during translational motion along $\mathrm{x}$-axis for configuration y $\left(0^{\circ}\right)$ is carried out for both sides $\mathrm{S}_{1}$ and $\mathrm{S}_{2}$. The energy minima for side 1 and side 2 are found to be $-0.6766 \mathrm{kcal} \mathrm{mole}^{-1}$ and $-0.1888 \mathrm{kcal} \mathrm{mole}^{-1}$, respectively. It is found out that the translational freedom is 
more prominent in in-plane translation than stacking. It can be seen from Figure 3 that this molecule has a preference for side $S_{1}$ and no definite preference for side $S_{2}$ is seen since it is constant over a longer translational region. The total interaction energy is constant over the translation region $-2 \pm 4 \AA$ for side $S_{1}$. This can be associated to the fluidity of the compound and is seen to maintain its alignment in mesophase.

\subsection{Configurational Probability Distribution in Stacking Mode}

The configurations with respect to translation have been only taken into account for probability estimation as no profound inclination has been observed with respect to rotation in terms of interaction energy. The probability variation with respect to translation along $\mathrm{x}$-axis during stacking mode for the configuration y $\left(0^{\circ}\right) \mathrm{z}\left(180^{\circ}\right)$ for faces 1 and 2 have been shown in Figures 4 and 5, respectively. The alteration of probability is found to be steady in the translation range $5 \pm 5 \AA$ for face 1 and $0 \pm 2 \AA$ for face 2 . This may be understood that gliding of a molecule one above the other is allowed for minute energy range. This can be associated to the fluidity of the compound and is seen to maintain its alignment in mesophase. Out of two faces, since face 2 exhibits lesser consistent translational region, it exhibits more stable stacked structure.

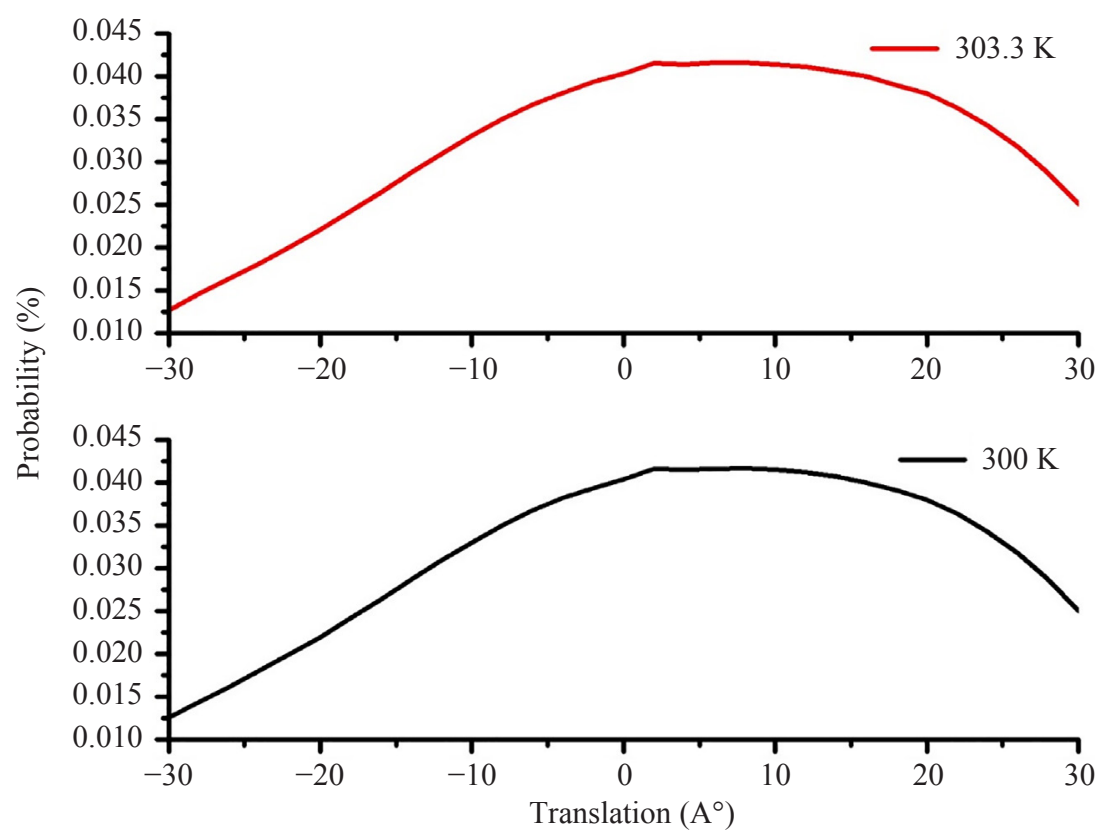

Figure 4: Variation of stacking probability with respect to translation along $\mathrm{x}$-axis for the configuration y $\left(0^{\circ}\right) \mathrm{z}\left(180^{\circ}\right)$ for face 1 . 


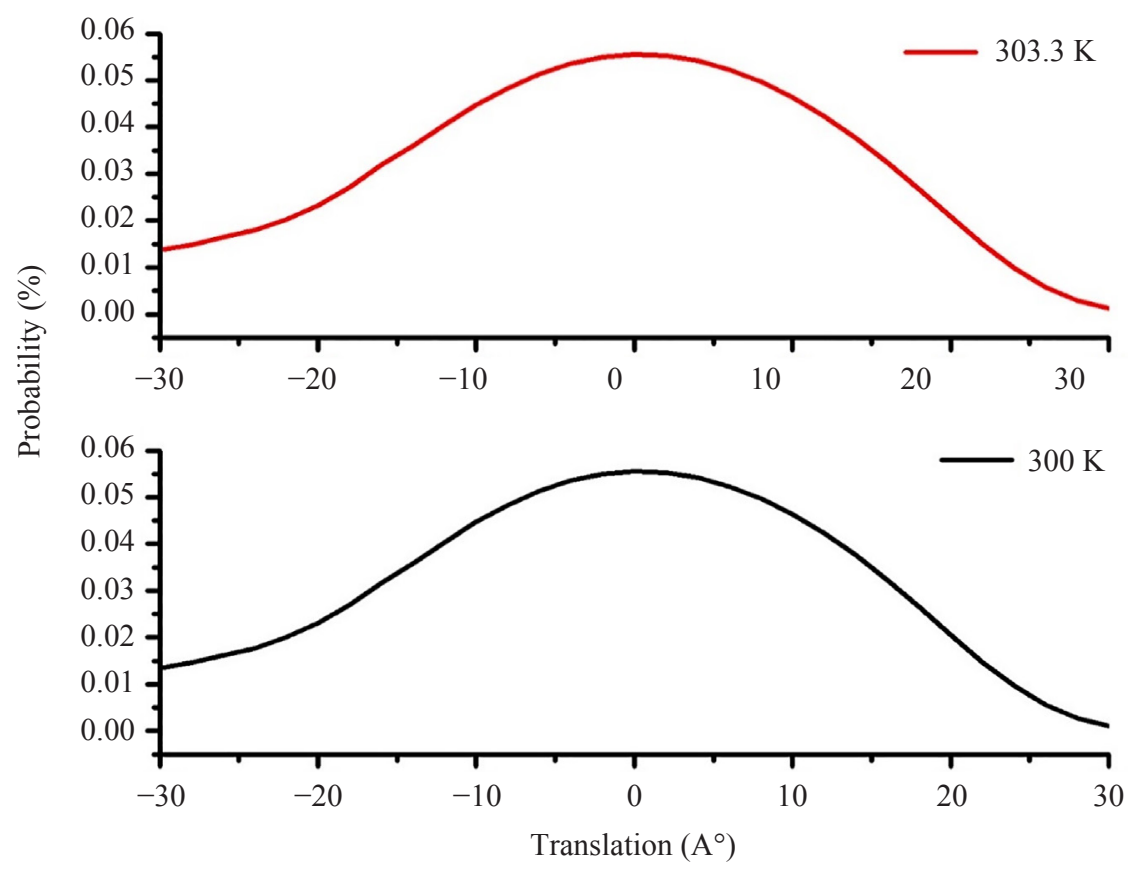

Figure 5: Variation of stacking probability with respect to translation along $\mathrm{x}$-axis for the configuration y $\left(0^{\circ}\right) \mathrm{z}\left(180^{\circ}\right)$ for face 2 .

On further refinement of the interacting configuration during translation and rotation of the molecules in the equilibrium condition, the energy has been minimised and the probability of the steady configuration is investigated with the consideration of the rotation of the molecule about $\mathrm{x}$-axis. Furthermore, the molecule has been rotated about z-axis for the configuration $\mathrm{x}\left(0^{\circ}\right)$ y $\left(0^{\circ}\right)$ and the probability variation is investigated at room and transition temperature. It has been found that a clear preference is obtained towards minimum energy point. The attained minimum energy is set as a beginning point and this procedure is further reiterated for smaller gaps. Translations and rotations were made about all the axes to minimise the energy. Translational accuracy of $0.1 \AA$ and $1^{\circ}$ of rotational accuracy is attained for a molecule with respect to other molecules. One should be very much conscious about the path of minimisation of energy depending upon the objective of computation and pre-selected condition.

\subsection{Configurational Probability Distribution in In-plane Mode}

The associating molecules are taken and constrained in the co-ordinate plane and the other one is constrained along the y-axis at an interspace of $8 \AA$ from the fixed one. From Figures 6 and 7, the probability variation in translational motion along 
$\mathrm{x}$-axis for the configuration $\mathrm{y}\left(0^{\circ}\right)$ shows that the translational freedom in in-plane interaction is more to stacking interaction with maximum probability at $26 \AA$. The probability is almost consistent in a region of translation $10 \pm 10 \AA$ for side 1 at both temperatures (Figure 6). The side 2 configuration exhibits approximately consistent region of $15 \pm 5 \AA$ at room temperature and $15 \pm 9 \AA$ at transition temperature (Figure 7). This larger region of no variation of probability offers sufficient stability for the molecules to attain the degree of order. This configuration that shows larger stable translational region has been refined for translation in $\mathrm{x}$-axis and the energy has been brought down at the equilibrium condition. Further, the probability variation has been studied and investigated with respect to rotation for the configuration y $\left(180^{\circ}\right)$. We observed that at a particular rotation point a high peak is obtained and is negligible at all other points. Therefore, the freedom with respect to rotation is more than stacking interaction. Now the molecules are rotated about $y$-axis and it is seen that there is no change in the configuration probability with rotation about $y$-axis with configuration $\mathrm{x}\left(0^{\circ}\right)$.

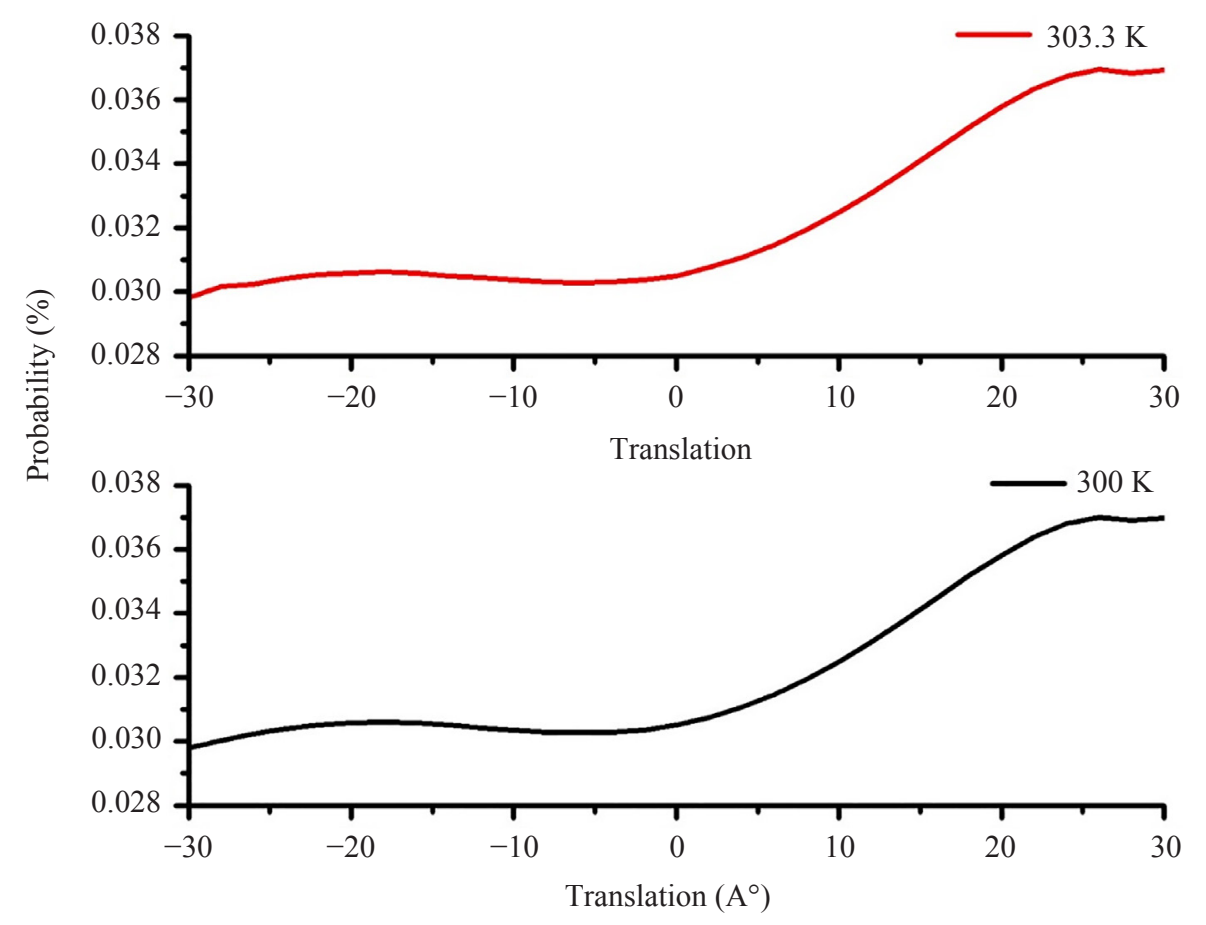

Figure 6: Variation of in-plane probability with respect to translation along $\mathrm{x}$-axis for the configuration y $\left(0^{\circ}\right)$ for side 1 . 


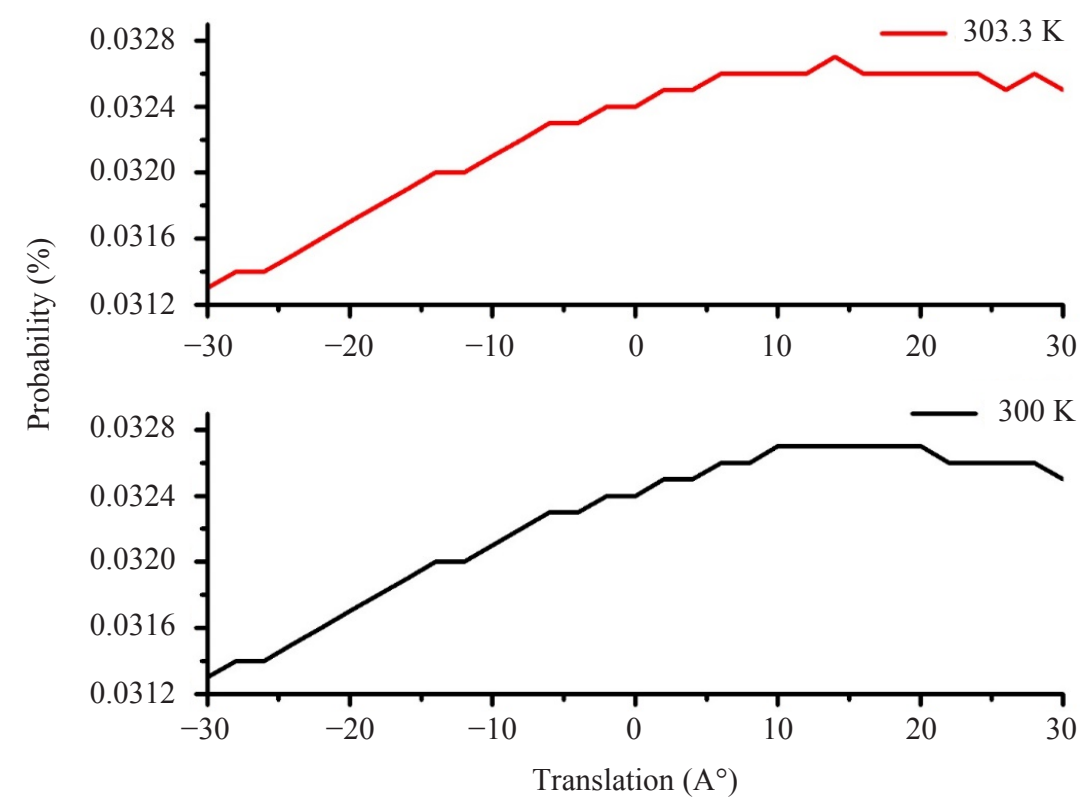

Figure 7: Variation of in-plane probability with respect to translation along $\mathrm{x}$-axis for the configuration y $\left(0^{\circ}\right)$ for side 2 .

\subsection{Configurational Probability Distribution in Terminal Mode}

These are the weakest among all modes of interactions. No specific preference has been noticed in terms of interaction energy and configurational probability.

\section{RIGIDITY EFFECT}

The different interaction modes will give different arrangements of molecules geometrically. The various interaction modes give information on different molecular arrangements inside the bulk materials. From the probability analysis, it has been noticed that during stacking mode, face 2 and side 1 during in-plane mode have preference of aligned structure of pairing of molecules. In general, it is clear that in an assembly of molecules every pair of molecules has a minimum energy configuration, i.e., a local minimum that is vital in case of close packing of the molecules. Although local minimum is of importance, the global minima are highly important. This is due to the fact that when the temperature is so high and the molecules are in completely disordered state then while cooling or coming back to the original configuration, there is a maximum probability for the molecules to occupy the positions with respect to global minima. The other minima follow the order, depending upon their own relative probabilities. 


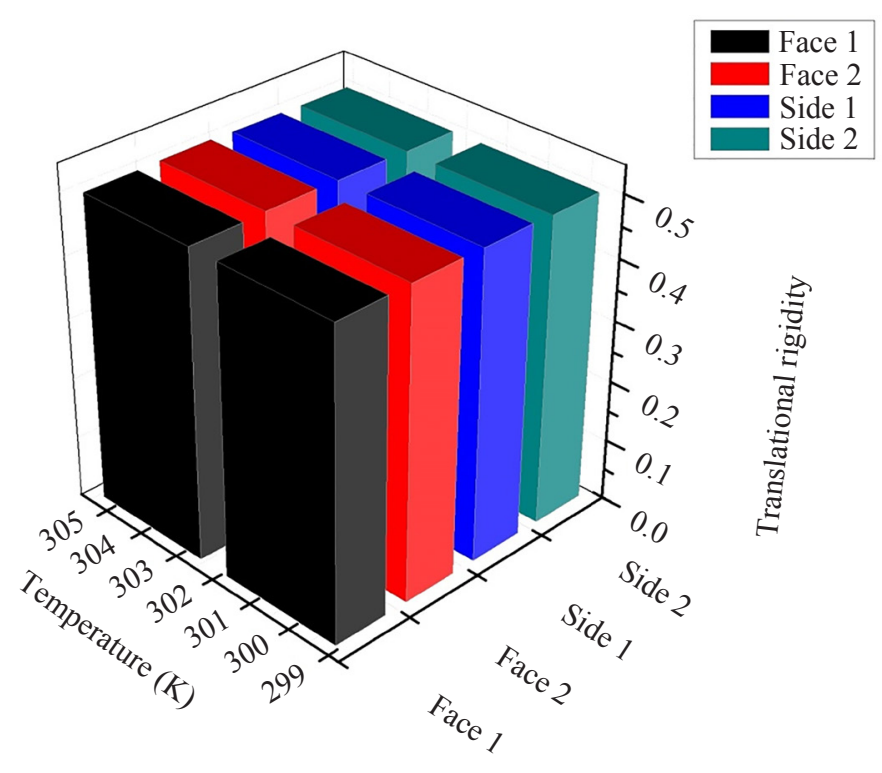

Figure 8: Translational rigidity as a function of temperature during stacking (face 1, face 2) and in-plane (side 1, side 2) at $300 \mathrm{~K}$ and $303.3 \mathrm{~K}$.

The nematic character of the liquid crystal is expressed by the translation control along the long molecular axis. The rigidity parameter has been estimated during stacking and in-plane modes for analysing the phase behaviour of the $3 \mathrm{CB}$ molecule at room and transition temperatures for face 1 , face 2 , side 1 and side 2 as shown in Figure 8. This has been defined as the ratio of probability being at maximum probable point having $\pm 0.2 \AA$ displacement along the long molecular axis. Along the long molecular axis, the translational rigidity is found to be 0.5018 and 0.5012 for face 1 , and 0.5040 and 0.5031 for face 2 at room temperature and transition temperature, respectively. This indicates that at low temperature the molecules are strongly bound but as the temperature increases the rigidity of the molecules decreases, i.e., the molecules obtain more freedom for translation or sliding upon other molecule. This seems to be more pronounced in in-plane interaction. For side 1 the rigidity value is 0.5020 and 0.5019 , and for side 2, 0.5002 and 0.5001 at room temperature and transition temperature, respectively. Upon comparison of face 2 and side 1 data (as these two exhibits minimum energy compared to other face and side), it may lead to a conclusion that the lesser translational rigidity is noticed during in-plane mode. This provides adequate flexibility for the molecules to align along long molecular axis. We can thus infer from the data that $S_{1}$ is the most preferable configuration which justifies the nematic character. 


\section{CONCLUSION}

The present theoretical view on cyanobiphenyl based molecule in polar aprotic solvent leads to the following conclusions:

1. The probability of various configurations during different modes of interaction with respect to translational motion at room and transition temperature has been calculated. The highest probability point shows the most stable configuration.

2. The rigidity parameter is useful in determination of flexibility of the molecule and helps in understanding the effect of the molecular motions on mesophase behaviour of the compound. Further, the chosen polar aprotic solvent has shown a strong impact on rigidity parameter that helps the molecule to exhibit higher stability and superior applications.

\section{REFERENCES}

1. Blinov, L. M. (2011). Structure and properties of liquid crystals. New York: Springer.

2. Pal, K. et al. (2015). Design, synthesis and application of hydrogen bonded smectic liquid crystal matrix encapsulated $\mathrm{ZnO}$ nanospikes. J. Mat. Chem. C, 3(45), 11907-11917. https://doi.org/10.1039/C5TC02436F

3. Asiya, S. I. et al. (2020). Reliable optoelectronic switchable device implementation by $\mathrm{CdS}$ nanowires conjugated bent-core liquid crystal matrix. Org. Elect., 82(1), 105592. https://doi.org/10.1016/j.orgel.2019.105592

4. Gudimalla, A. et al. (2020). Nanoparticle-stabilized lattices of topological defects in liquid crystals. Int. J. Thermophys., 41(4), 51-67. https://doi.org/10.1007/ s10765-020-02631-w

5. Jose, T. J. et al. (2019). Quantum and thermodynamic estimation of mesostate behaviour of alkyl benzoic acids in dielectric medium. Arab. J. Sci. Eng., 44(7), 6601-6608. https://doi.org/10.1007/s13369-019-03791-0

6. Kumar, S. (2020). Investigations on discotic liquid crystals. Liq. Cryst., 47(8), 1195-1203. https://doi.org/10.1080/02678292.2020.1720840

7. Das, P. \& Lakshmi Praveen, P. (2019). Solvent polarity and end chain length effects in thermotropic mesophase formation process: Comparative quantum and thermotropic analysis. J. Mol. Liq., 288(1), 1-7. https://doi.org/10.1016/ j.molliq.2019.111029

8. Prasad, S. \& Ojha, D. P. (2019). Computational study of molecular geometries, and vibrational characteristics of two liquid crystalline disubstituted biphenylcyclohexanes using ab-initio methods. Mol. Cryst. Liq. Cryst., 682(1), 27-43. https://doi.org/10.1080/15421406.2019.1655975 
9. Sánchez, E. G. et al. (2002). Predicting the phase diagram of a liquid crystal using the convex peg model and the semiempirical PM3 method. J. Phys. Chem. A, 106(43), 10342-10349. https://doi.org/10.1021/jp021453o

10. Pople, J. A. \& Beveridge, D. L. (1970). Approximate molecular orbital theory. New York: Mc-Graw Hill.

11. Cestari, M. (2011). Phase behavior and properties of the liquid-crystal dimer 1",7"-bis(4-cyanobiphenyl-4'-yl) heptane: A twist-bend nematic liquid crystal. Phys. Rev. E, 84(3), 1-20. https://doi.org/10.1103/PhysRevE.84.031704

12. Lakshmi Praveen, P. \& Ojha, D. P. (2011). Phase organization in laterally substituted dicyclohexylethane derivatives: A molecular model for smectogens. J. Mol. Liq., 161(1), 44-48. https://doi.org/10.1016/j.molliq.2011.04.008

13. Lakshmi Praveen, P. \& Ojha, D. P. (2011). Role of molecular rigidity on phase organization of a smectic liquid crystal: A theoretical model. Mat. Chem. Phys., 126(1-2), 248-252. https://doi.org/10.1016/j.matchemphys.2010.11.031

14. Haase, W. et al. (1983). Solid state polymorphism in 4-Cyano-4'$n$-propylbiphenyl and X-ray structure determination of the higher melting modification. Mol. Cryst. Liq. Cryst., 100(3-4), 211-221. https://doi.org/10 $.1080 / 00268948308075353$

15. Pal, K. et al. (2013). Switching of ferroelectric liquid crystal doped with cetyltrimethylammonium bromide-assisted $\mathrm{CdS}$ nanostructures. Nanotechnol., 24(12), 125702. https://doi.org/10.1088/0957-4484/24/12/125702

16. Pal, K. et al. (2019). Soft, self-assembly liquid crystalline nanocomposite for superior switching. Electron. Mater. Lett., 15(1), 84-101. https://doi.org/10.1007/ s13391-018-0098-y

17. Pullmann, B. (ed.). (1978). Intermolecular interactions: From diatomic to biopolymers. New York: John Wiley.

18. Das, P. \& Lakshmi Praveen, P. (2017). Probabilistic and thermodynamic approach on phase behavior of nematic liquid crystals: A comparative study. Mol. Cryst. Liq. Cryst., 652(1), 185-194.

19. Kitaygorodsky, A. I. (1961). The interaction curve of non-bonded carbon and hydrogen atoms and its application. Tetrahedr., 14(3-4), 230-236. https://doi. org/10.1016/S0040-4020(01)92172-6

20. Lakshmi Praveen, P. (2018). 7-Hexyloxy-3-[4'-(3-methylbutyloxy) phenyl]-4H1-benzopyran-4-one: Study of Smectic behaviour and UV absorption profile. Mol. Cryst. Liq. Cryst., 667(1), 44-53. https://doi.org/10.1080/15421406.2018 .1553755

21. Rowlinson, J. S. (2005). The Maxwell-Boltzmann distribution. Mol. Phys., 103(21-23), 2821-2828. https://doi.org/10.1080/002068970500044749 